\title{
Marco de referencia para la planificación de generación distribuida en zonas no interconectadas
}

\section{Framework for distributed generation planning troughout non- connected areas}

\author{
Gabriel Jaime Correa-Henao \\ Ph.D. en Ingeniería Eléctrica \\ Fundación Universitaria Luis Amigó \\ Medellín, Colombia \\ gabriel.correahe@amigo.edu.co
}

\author{
Juan Carlos Rojas-Zerpa \\ Ph.D. en Energías Renovables \\ Universidad de los Andes \\ Mérida, Venezuela \\ juancrojas@ula.ve
}

\begin{abstract}
Resumen- Se presenta una propuesta metodológica del diseño, desarrollo y aplicación para el apoyo a la toma de decisiones multicriterio (MCDM, por sus siglas en inglés) que permita planear un sistema de suministro eléctrico, teniendo en cuenta variables de impulso al desarrollo local de pequeñas poblaciones rurales 0 remotas, específicamente a través de un caso de estudio en los Llanos Orientales de Colombia. Para el efecto, se propone el uso de dos técnicas bien documentadas, como el Proceso Analítico Jerárquico (AHP, por sus siglas en inglés) y el método VIKOR, al tiempo que se aporta un cambio de paradigma en la planificación de los sistemas de generación distribuida, considerando para ello la inclusión integral de aspectos técnicos, económicos, sociales y ambientales. En esta propuesta, los decisores pueden observar comparativamente el comportamiento de los sistemas de suministro eléctrico, ya sea mediante la generación distribuida o mediante la extensión de las redes eléctricas.
\end{abstract}

Palabras clave- Generación distribuida, toma de decisiones, análisis multiobjetivo, AHP, VIKOR

\begin{abstract}
The authors present a suitable methodology to support designing, development and implementation in a Multicriteria Decision Making (MCDM) environment. This is done in order to perform planning of a power supply system that takes into account variables that boost local development of small rural or remote areas, specifically through a case study in the eastern plains of Colombia. The use of two well-known techniques such as Analytic Hierarchy Process (AHP) and VIKOR techniques are applied as a new method to develop planning of distributed generation systems. Such method considers the inclusion of technical, economic, social and environmental aspects in order to guide planning tasks. In this proposal, decision makers are able to compare the performing of power systems, by either investing in electric distribution grid extension or by installing distributed generation electric systems.
\end{abstract}

Keywords- Distributed generation, decision making, multiobjective analysis, AHP, VIKOR

\section{INTRODUCCIÓN}

La energía eléctrica es el motor de desarrollo que permite evolucionar a la humanidad como civilización, y también proporciona la clave de su equilibrio con el entorno natural. Es la carta de la desigualdad entre mundos sociales injustamente diferenciados [1]. La electricidad está presente en todos los sectores económicos y sociales, gracias a su gran variedad de aplicaciones, que van desde el alumbrado, calefacción o ventilación, hasta el campo de las telecomunicaciones, tecnologías de la información, procesos industriales, etc. [2] En el actual escenario energético, se consolida la creciente utilización de la energía eléctrica, frente a fuentes primarias como los combustibles fósiles, las tecnologías de los sistemas de generación eléctrica distribuida, así como los sistemas de generación híbrida activa, lejos de conducirnos a una meta considerada generalmente como utópica, establece las bases de un camino mediante la cual se podrá alcanzar su desarrollo y evolución en condiciones mucho más favorables que las que en la actualidad tenemos, en el escenario de energía centralizada [3], [4].

En esta contribución técnica se presenta la aplicación de un desarrollo metodológico para la planificación de generación distribuida (GD), soportado en métodos de análisis o ayuda a la decisión multicriterio (Multicriteria Decision Making, MCDM, por sus siglas en inglés) para facilitar la selección de un sistema de suministro eléctrico que aporte mayores beneficios al desarrollo local de pequeñas poblaciones rurales o remotas de países en vías de desarrollo, utilizando para ello 
dos técnicas, tales como: el Proceso Analítico Jerárquico (Analytic Hierarchy Process, AHP, por sus siglas en inglés) [5] y el método VIKOR [6-8], una extensión de la Programación Compromiso [9][11]. De esta manera, se realizan aportes dentro de los paradigmas de planificación en sistemas de GD, considerando para ello la inclusión integral de aspectos técnicos, económicos, sociales y ambientales, lo cual se valida mediante un caso de estudio para la energización de comunidades organizadas en los Llanos Orientales de Colombia, a partir de un ordenamiento de soluciones compromiso consideradas (alternativas).

La metodología presentada en esta contribución técnica se fundamenta en el diseño óptimo de instalaciones eléctricas de GD, la cual se estima a partir de modelos de configuración proporcionados por la herramienta informática i-Hoga [12], lo cual permite documentar la experiencia derivada de la aplicación de tecnologías de GD, en aspectos requeridos dentro de zonas no interconectadas (ZNI) de diferentes regiones de Colombia, con aplicaciones en soluciones energéticas considerando aspectos técnicos, económicos, sociales y ambientales, para observar comparativamente el comportamiento de los sistemas de suministro eléctrico.

Este artículo se compone de las siguientes secciones: la primera sección presenta el marco teórico en torno a la propuesta metodológica para planificación de sistemas GD en ZNI. En la segunda parte del artículo se presenta la realización de una metodología de dimensionamiento, con aplicación a un caso de estudio en una ZNI de Colombia. Lo anterior conduce a la presentación de algunas conclusiones, la tercera y última parte del artículo, donde se demuestra la ventaja económica de implementar las instalaciones aisladas, en vez del tradicional modelo de extensión de red de distribución rural.

\section{CONCEPTOS Y DESARROLLOS EN GENERACIÓN DISTRIBUIDA}

La definición en el concepto de generación distribuida (GD) no es única. En el contexto del presente trabajo, se entenderá por generación distribuida el proceso de producción y uso de energía eléctrica a pequeña o mediana escala (desde algunos cientos de $\mathrm{W}$ hasta los $10 \mathrm{MW}$ ), con una cercanía a los centros finales de consumo y con posibilidad de interactuar con las redes de interconexión eléctrica [13]. A continuación se discuten los aspectos inherentes a la conceptualización de la generación centralizada y distribuida, atendiendo de igual manera otros conceptos como generación integrada, dispersa y descentralizada:

\subsection{Generación eléctrica centralizada (GEC)}

La clásica cadena de suministro puede ser resumida de la siguiente manera: primero la energía primaria está disponible remotamente para ser transportada hasta las grandes plantas donde efectivamente puede ser transformada, luego de la transformación en un vector energético se vuelve a transportar hasta los sistemas de distribución, que finalmente entregaran la energía a los usuarios. De esta manera, la GEC cuenta con unidades generadoras entre $100 \mathrm{MW}$ y $1 \mathrm{GW}$ de potencia [14], las cuales están localizadas generalmente lejos de los centros de carga, en aquellos lugares donde los recursos naturales están disponibles (principalmente fósiles e hidroenergía de gran escala).

\subsection{Generación descentralizada}

La generación descentralizada corresponde a un término que cada vez toma mayor uso, cuya definición tiene dos connotaciones diferentes. Según la Agencia Internacional de la Energía [15] se refiere a la generación descentralizada como un sistema de recursos energéticos distribuidos (DER, siglas en inglés) conectados a una red de distribución. En contraste, en [16] este tipo de generación se refiere a las unidades de producción de energía que son autónomas, en la cual no hay interacción con otras unidades o sistemas. Por su parte, en [17] se explica que: "todos los sistemas descentralizados son distribuidos, pero un sistema distribuido no necesariamente es descentralizado".

\subsection{Generación distribuida (GD)}

El principal objetivo de los sistemas de GD corresponde al suministro de electricidad en regiones distantes de las redes de distribución, y en las comunidades no conectadas donde los costos de la transmisión o distribución mediante la red son demasiado altos [18] Estos sistemas de ge- 
neración están en auge y son objeto de amplios estudios en una variedad de disciplinas de la ciencia, la ingeniería y en los desarrollos tecnológicos y comerciales.

La discusión del término GD es consecuencia del interés que ha suscitado esta nueva tendencia dentro de la industria de la energía eléctrica. Parece ser que primero han surgido las aplicaciones y luego se ha comenzado a trabajar en la conceptualización de esta forma de producción y aprovechamiento de energía [19].

No obstante, teniendo en cuenta que la generación distribuida está más cerca de los usuarios, es evidente el vínculo existente entre fuente de energía, usuarios y localización geográfica. Un aspecto importante relacionado con el tamaño de la generación distribuida, específicamente para aplicaciones rurales, se refiere a las aportaciones disponibles en [17], donde se establece que el tamaño de la generación distribuida oscila entre 1 y 100 kW. Los sistemas de pequeña potencia describen las aplicaciones puntuales como una vivienda, un sistema de bombeo de agua, entre otros; los sistemas de mayor tamaño se vinculan con centros de carga más intensivos, tales como: un grupo de viviendas, un centro poblado, etc.

El tamaño de la generación distribuida también es importante en atención al punto de evacuación de la energía a la red (para los sistemas GD conectados a red) o para la configuración de la relación oferta-demanda de un centro poblado, aldea o caserío. Estos sistemas pueden ser combinados con un sistema de gestión y almacenamiento de energía con la finalidad de mejorar la operación del sistema de distribución [20]. En la Tabla I se muestra un compendio de los rangos de operación de GD, cuya potencia de generación se relaciona directamente con la tecnología empleada.

TABLA I

TAMAÑO DE LA GENERACIÓN DISTRIBUIDA EN FUNCIÓN DE LA TECNOLOGÍA DE GENERACIÓN

\begin{tabular}{|c|c|}
\hline Tecnología & Rango de aplicaciones \\
\hline Turbinas de gas de ciclo combinado & $1-20 \mathrm{MW}$ \\
\hline Motores de combustión interna (gasóleo) & $20 \mathrm{~kW}-10 \mathrm{MW}$ \\
\hline Motores de combustión interna (gas) & $5 \mathrm{~kW}-5 \mathrm{MW}$ \\
\hline Turbina de combustión & $1-250 \mathrm{MW}$ \\
\hline Microturbinas & $30 \mathrm{~kW}-1 \mathrm{MW}$ \\
\hline Pequeña hidroenergía & $1-100 \mathrm{MW}$ \\
\hline Micro hidroenergía & $25 \mathrm{~kW}-1 \mathrm{MW}$ \\
\hline Turbina eólica & $200 \mathrm{~W}-3 \mathrm{MW}$ \\
\hline Solar fotovoltaica & $20 \mathrm{~W}-100 \mathrm{~kW}$ \\
\hline Solar térmica (captador central) & $1-10 \mathrm{MW}$ \\
\hline Biomasa (gasificación) & $100 \mathrm{~kW}-20 \mathrm{MW}$ \\
\hline Pilas de combustible de ácido fosfórico (PAFC) & $200 \mathrm{~kW}-2 \mathrm{MW}$ \\
\hline Pilas de combustible de carbonatos fundidos (MCFC) & $50 \mathrm{~kW}-2 \mathrm{MW}$ \\
\hline Pilas de combustible polimérica (PEMFC) & $1 \mathrm{~kW}-250 \mathrm{~kW}$ \\
\hline Pilas de combustible de óxido sólido (SOFC) & $1 \mathrm{~kW}-5 \mathrm{MW}$ \\
\hline Geotérmica & $5-100 \mathrm{MW}$ \\
\hline Energía del mar & $100 \mathrm{~kW}-1 \mathrm{MW}$ \\
\hline
\end{tabular}

Fuente: los autores. (Ackermann, Andersson, \& Söder, 2001), (Pepermans, Driesen, Haeseldonckx, Belmans \& D’haeseleer, 2005) 
La implementación de los sistemas GD puede ser exitosa si las políticas de los estados se orientan adecuadamente por los interesados. Además de los gobiernos, hay que destacar el rol que pueden jugar los empresarios, los consumidores, las agencias internacionales y las organizaciones no gubernamentales (ONG), para contribuir a la democratización de la energía. En general, las tecnologías de generación distribuidas de origen renovable, al ser aplicadas en emplazamientos que poseen potenciales recursos energéticos (energía local), efectivamente pueden aproximarse al cumplimiento de aspectos tecnológicos, económicos, sociales y ambientales requeridos en un sistema de suministro energético en zonas no interconectadas [18].

\subsection{Aspectos importantes sobre planificación de la energía}

Al definir el marco de actuación para conseguir una buena distribución o asignación de los recursos de un sistema, es conveniente situar cada actividad por realizar dentro de un esquema general de planificación. Dado que a veces, o generalmente, la planificación puede tratarse de un proceso de toma de decisiones; se pueden distinguir varias etapas [23]:

- Identificación del problema.

- Desarrollo de alternativas.

- Elección de la alternativa más conveniente.

- Ejecución del plan.

Para el efecto, se suelen diseñar modelos fundamentados en investigación de operaciones, los cuales se pueden simplificar, pero que requieren el uso de datos confiables. La planificación de la energía implica encontrar un conjunto de fuentes y equipos de conversión que satisfagan los requerimientos de energía de todas las tareas (demanda) de una manera óptima, lo cual podría ser al nivel centralizado o descentralizado [24].

Por tal motivo, el suministro de energía eléctrica en zonas no interconectadas de un país como Colombia, puede ser calificado como una actividad compleja que trasciende a la simple solución de la mejor tecnología. La literatura especializada en el área de planificación energética en regiones rurales y aisladas está bien documentada y sirve como base teórica para guiar la toma de decisio- nes. Particularmente se destaca la información contenida en artículos como [18], [25].

\section{FUNDAMENTOS DE HERRAMIENTAS PARA LA TOMA DE DECISIONES MULTICRITERIO}

La planificación coherente de la energía descentralizada implica la apreciación de múltiples factores, considerados en un proceso de decisión multidimensional como aspectos técnicos, económicos, ambientales y sociales, teniendo en cuenta las condiciones y limitaciones del entorno, para favorecer a las poblaciones locales (rurales y remotas) en el acceso a la energía eléctrica[19]. En este contexto, y dado que la planificación de los sistemas de suministro de energía representa un problema multidimensional, su expresión matemática es de naturaleza multicriterio-multiobjetivo o viceversa. Basados en estos hallazgos, el análisis de decisiones multicriterio es un método conveniente para tales fines [26].

Para efectos ilustrativos en la aplicación de la propuesta metodológica, se proporciona una breve descripción de dos herramientas que soportan la toma de decisiones multicriterio.

\subsection{Proceso analítico jerárquico (AHP, por sus siglas en inglés)}

AHP es una poderosa herramienta que puede ser usada para la descomposición de problemas más complejos en un modelo o nodo jerárquico [5], [27]. El propósito del método es permitir que el agente decisor pueda estructurar un problema multicriterio en forma visual, mediante la construcción de un modelo jerárquico que básicamente contiene tres niveles: meta u objetivo, criterios y alternativas [23]. Una vez construido dicho modelo jerárquico, se realizan comparaciones de pares entre dichos elementos (criterios - subcriterios y alternativas) y se atribuyen valores numéricos a las preferencias señaladas por las personas, entregando una síntesis de las mismas mediante la agregación de esos juicios parciales.

La metodología AHP se ha implementado computacionalmente, es muy popular y tiene amplia utilización como una herramienta efectiva en el apoyo a la toma de decisiones, según se puede consultar en [28-30]. 


\subsection{Optimización multicriterio y solución compromiso (VIKOR)}

El desarrollo del método VIKOR se documenta inicialmente en [7], [8], como estrategia para la optimización multicriterio de sistemas complejos, de tipo discreto, con criterios conflictivos y no cuantificables. En dicho método se determina una clasificación de soluciones compromiso, la solución compromiso y la verificación de la estabilidad en la toma de decisiones. El índice del ordenamiento multicriterio obtenido está basado en la medida particular de proximidad a la solución ideal. Asumiendo que cada alternativa es evaluada acorde con cada función de criterio, el ordenamiento de soluciones compromiso puede obtenerse por comparación a la medida de proximidad a la alternativa ideal [8]. La medida multicriterio del ordenamiento de soluciones compromiso proviene de la métrica $L_{p}$, la cual es usada como una función de agregación en un método de la programación compromiso, que se calcula según (1):

$$
\begin{aligned}
& L_{p, j}=\left\{\sum_{i=1}^{n}\left[\frac{w_{i}\left(f_{i}^{*}-f_{i j}\right)}{\left(f_{i}^{*}-f_{i}^{-}\right)}\right]^{p}\right\}^{1 / p}, \\
& \text { donde } 1 \leq p \leq \infty ; j=1,2, \ldots, J ; \\
& f_{i}^{*}=\operatorname{má} x\left(f_{i j}\right) ; f_{i}=\operatorname{mí} n\left(f_{i j}\right)
\end{aligned}
$$

fij representa la i-ésima función de un criterio de beneficio.

La solución compromiso obtenida podría ser aceptada por los decisores, ya que esta provee una máxima utilidad de grupo (representada por un valor mínimo de $S$ ) de la mayoría, y un mínimo de rechazo individual (representado por un valor mínimo de $R$ ) de los oponentes. La solución compromiso podría ser la base de las negociaciones, involucrando las preferencias de los decisores por la ponderación de los criterios [8].

En esta contribución técnica se propone el uso de una versión mejorada de la metodología, conocida como VIKOR Comprensivo [31], correspondiente a una técnica para el tratamiento de problemas multiobjetivo que impliquen la selección de una solución entre en grupo finito de alternativas, razón por la cual se propone como la herramienta de cálculo de la metodología propuesta, además de contar con compatibilidad para combinarse con otro método discreto como AHP. EI procedimiento de implementación se describe a continuación.

\subsubsection{Normalización de datos de entrada}

La normalización propuesta por[31] debe realizarse mediante (2) y (3).

$$
\begin{gathered}
A_{i j}=\left|\left(r_{i j}-T_{j}\right)\right| \times\left(-A_{j}\right)^{-1} \\
A_{j}=r_{j}^{m a ́ x}-r_{j}^{\min }
\end{gathered}
$$

$A_{i j}=$ Valor normalizado de la alternativa $i$ respecto al criterio $\mathrm{j}$.

$$
r_{i j}=(i=0,1,2,3, \ldots, m),(j=0,1,2,3, \ldots, n),
$$
corresponden a los elementos de la matriz de decisión (alternativa $i$, respecto al criterio $j$ ).

$T_{j}=$ Valor ideal o valor objetivo de rij para todo criterio $j\left(T_{1}, T_{2}, T_{3}, \ldots, T_{j}, \ldots, T_{n}\right)$.

$r_{j}^{\text {máx }}, r_{j}^{\text {min }}=$ Máximo y mínimo valor en el criterio $j$ respectivamente.

Tanto AHP como VIKOR representan dos técnicas de Análisis Multiobjetivo que perfectamente se pueden combinar para el tratamiento de problemas de naturaleza discreta, como es el caso que compete a la planificación de sistemas de suministro eléctrico descentralizado. Los diferentes atributos de estas técnicas, potencialmente, aportan elementos para ofrecer una herramienta de fácil aplicación, amplia transparencia, apropiada para la participación de decisores y de credibilidad en los resultados.

\subsubsection{Cálculo de los parámetros de $S_{i}, R_{i} y Q_{i}$}

Una vez se obtienen los términos de la matriz $A_{i j}$ normalizada (2), se debe identificar la naturaleza de los valores o métricas $\mathrm{R}, \mathrm{S}, \mathrm{Q}$ requeridos en la solución compromiso más eficiente dentro del problema de planificación[26].

$$
\begin{gathered}
S_{i}=\sum_{i=1}^{n} w_{j} \times\left(1-e^{-A_{i j}}\right) \\
R_{1}=\operatorname{má}_{j}\left(w_{j} \times\left(1-e^{-A_{i j}}\right)\right) \\
Q_{1}=\left[\left(S_{i}-S^{-}\right) /\left(S^{+}-S^{-}\right)\right] \cdot v+ \\
{\left[\left(R_{i}-R^{-}\right) /\left(R^{+}-R^{-}\right)\right] \cdot(1-v)}
\end{gathered}
$$

$S_{i}$ : Se refiere a la medida de utilidad o total satisfacción. 
$R_{i}$ : Se refiere a la medida de pesar o insatisfacción.

$Q_{i}$ : Representa el valor de VIKOR para la i-ésima alternativa.

$S^{-}, S^{+}$: Valor mínimo y máximo de $S_{i}$; respectivamente.

$R^{+}, R^{*}$ : Valor máximo y mínimo de $R_{i}$; respectivamente.

$v$ : Es el peso de la estrategia asociada a la máxima utilidad del grupo.

$1-v$ : Es el peso del mínimo rechazo individual de los oponentes.

El peso de la estrategia " $v$ " toma valores en el rango entre 0 y 1 . Un valor de " $v$ " $=0.5$ implica una estrategia de consenso entre ambas posturas; si " $v$ " $>0,5$ la mayoría decide, por el contrario si " $v$ " $<0,5$ la minoría decide (veto). Los resultados de $S_{i}, R_{i}$ y $Q_{i}$ son tres listas de clasificación (o ranking) ordenados en forma decreciente.

\subsubsection{Verificación de la estabilidad de las soluciones ordenadas de compromiso}

Para esta verificación se debe proponer como mejor solución compromiso a la alternativa $\left(A^{(I)}\right)$, la cual es la solución mejor posicionada en el ordenamiento de soluciones de la métrica $Q$ (valor de VIKOR), sí y solo si las siguientes dos condiciones se satisfacen.

\section{Condición 1: Ventaja aceptable:}

$$
\begin{aligned}
& Q\left(A^{(2)}\right)-Q\left(A^{(1)}\right) \geq D Q \\
& D Q=1 \times(M-1)^{-1}
\end{aligned}
$$

$A^{(2)}$ : Es la segunda mejor solución compromiso (alternativa) del ranking $Q$.

$D Q$ : Relación de comparación que es función del número de alternativas.

$M$ : Número de alternativas.

Condición 2: Estabilidad aceptable en la toma de decisiones:

La alternativa $A^{(l)}$ también debería ser la alternativa mejor posicionada en el ranking de la métrica $S$ (medida de satisfacción) y/o $R$ (medida de pesar o insatisfacción). De lo contrario, debe proponerse un conjunto de soluciones compromiso si una de las condiciones no se satisface. Para ello, se pueden dar las siguientes opciones:
- Si la condición $C 2$ no se satisface, $A^{(1)}$ y $A^{(2)}$ serán las mejores soluciones, o

- Si $C 1$ no se satisface, entonces las mejores soluciones serán $A^{(1)}, A^{(2)}, \ldots, A^{(M)}$.

- Donde $A^{(M)}$ se determina mediante la relación proporcionada por (9):

$$
Q\left(A^{(M)}\right)-Q\left(A^{(1)}\right) \leq D Q
$$

\section{CASO APLICATIVO EN PLANIFICACIÓN ENERGÉTICA DE UNA ZONA NO INTERCONECTADA}

En correspondencia al planteamiento de las alternativas de suministro eléctrico descentralizado, se verificará la incorporación de un proceso de optimización multiobjetivo mediante algoritmos genéticos para el dimensionado de las fuentes de generación, lo cual requerirá el uso de i-HOGA, un software de uso libre [12]. La metodología propone el uso secuencial de las técnicas de decisión multicriterio AHP y VIKOR, que serán aplicadas posteriormente a un caso real que consiste en la selección de la mejor propuesta de suministro eléctrico para una zona no interconectada, ubicada en una zona de los Llanos Orientales de Colombia.

En este caso, AHP se utilizará para establecer la ponderación de preferencia que los decisores asignarán a cada uno de los criterios y subcriterios considerados en la toma de decisiones. Para la selección de las alternativas se utilizará el método VIKOR, el cual permitirá generar una clasificación de soluciones compromiso en función de los criterios y ponderaciones tomadas en cuenta para tal propósito.

\subsection{Caracterización del contexto geográfico y físico-natural de la ZNI}

Para el caso de estudio se ha optado por cubrir los requerimientos energéticos en 4 haciendas ubicadas en la vereda "Gaviotas" del municipio de Puerto Lleras (departamento del Meta, Colombia) enfocado en aplicaciones agropecuarias para una zona no interconectada (Llanos Orientales de Colombia), en cuyo caso particular, existen dificultades para acceder al agua superficial, pues la región suele sufrir de fuertes sequías. En dichas haciendas se destina el uso de suelo para faenas pecuarias y para la siembra de palma africana. 
Se puede consultar la Fig. 1 para establecer la localización geográfica de la zona en la que se realiza el estudio de planificación para el suministro energético. Las haciendas están ubicadas en la orilla de una carretera rural, a lo largo de $26 \mathrm{~km}$. La distancia media entre cada una de las casas donde residen los administradores y propietarios de las haciendas es de $6.5 \mathrm{~km}$.

\subsection{Caracterización de las necesidades actuales y futuras de electricidad}

La caracterización de aplicaciones está dirigida a identificar el uso de las fuentes de energías primarias o combustibles y su razón de uso para la cocción de alimentos, agua caliente sanitaria, iluminación y electricidad para la utilización de dispositivos eléctricos como el teléfono, televisor, radio, entre otros. También es conveniente precisar las expectativas de las futuras necesidades energéticas a mediano y largo plazo.

Para la determinación de las soluciones eficientes en generación distribuida (Dispersa GDD- o Compacta -GDC-) [32], es necesario aplicar un software de optimización multiobjetivo que permita generar soluciones óptimas desde varias dimensiones, por ejemplo, desde el punto de vista económico (mínimo coste) y ambiental (mínimas emisiones de $\mathrm{CO}_{2}$ ). Adicionalmente, se considera el perfil de carga de cada una de las casas ubicadas en las haciendas agropecuarias. Estos requerimientos están relacionados con las curvas de carga objetivo para satisfacer la demanda de energía eléctrica prevista a largo plazo (año de diseño), que en este caso se proyecta a 40 años, correspondiente a la vida útil de una red de distribución de bajo voltaje (13.6kV/0.22kV).

La demanda final de energía se ha calculado, teniendo en cuenta tres aspectos: las necesidades o aspiraciones de aplicaciones domésticas (se fijó una cantidad mínima de energía que actualmente sirve un sistema de electrificación rural conectado a red, en Colombia), el crecimiento natural de la población y el crecimiento en el consumo por uso de la electricidad, la necesidad de atender gastos energéticos propios de las faenas agropecuarias en la región geográfica (bombeo de agua, energización de cercas, iluminación, refrigeración, climatización y confort rural). La Fig. 2 proporciona un perfil de la curva de carga para cada una de las haciendas que hace parte del estudio, donde se puede observar que las horas de mayor consumo de energía ocurren al principio de la mañana y al final de la tarde, coincidiendo con las costumbres de alimentación y organización del hogar. Adicionalmente, en la Tabla II se proporciona un análisis detallado de los aplicativos requeridos en virtud de la energización de cada una de las haciendas que hacen parte del estudio.

En este sentido, el principal elemento que determina la demanda se ha fijado de acuerdo con la cantidad de energía que puede servir una red eléctrica rural convencional, lo cual oscila entre $1 \mathrm{kWh}$ y $10 \mathrm{kWh}$ por vivienda diariamente, para un hogar en clima cálido. En efecto, este valor es muy superior a lo estipulado por las metas de milenio en lo referente a la cantidad mínima de energía que se debería disponer para superar la pobreza, es decir, $250 \mathrm{kWh}$ por vivienda y año [33].

Algunos datos relevantes, requeridos para la construcción del modelo de optimización, con información importante respecto a las condiciones del Sistema Interconectado Nacional (SIN), así como proyecciones en los consumos de las viviendas rurales, se muestran en la Tabla III. La curva de carga de diseño resultante, para cada una de las viviendas que componen las haciendas bajo estudio, se muestra en la Fig. 2.

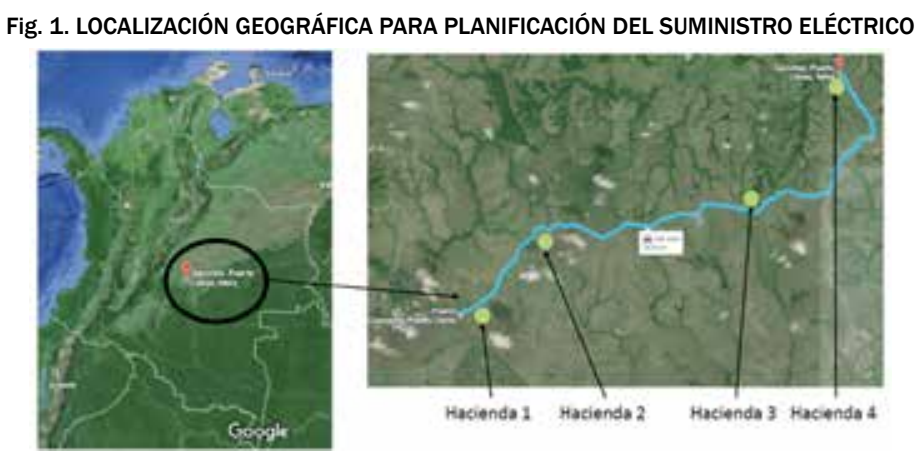

Fuente: Google-Earth. 
Fig. 2. PERFIL DE CARGA HORARIA PARA CADA VIVIENDA QUE REQUIERE ENERGIZACIÓN, CON PROYECCIÓN DE DISEÑO A 40 AÑOS

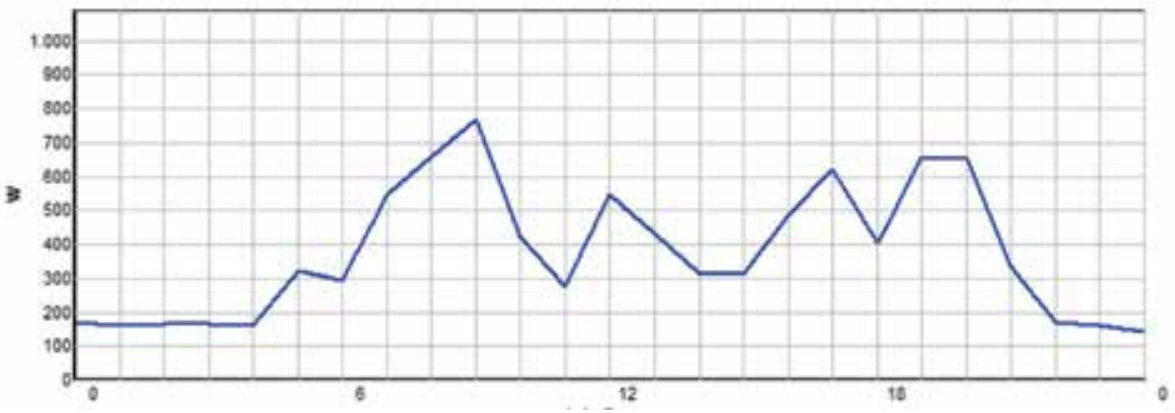

Fuente: los autores.

TABLA II

REQUERIMIENTOS ENERGÉTICOS ACTUALES PARA AUTOCONSUMO Y FAENAS AGROPECUARIAS

\begin{tabular}{|c|c|c|c|c|c|c|}
\hline \multicolumn{5}{|c|}{ TOTAL ENERGÍA DIARIA EN DC REQUERIDA EN INSTALACIÓN } & 190 & [Wh/día] \\
\hline \multicolumn{5}{|c|}{ TOTAL ENERGÍA DIARIA EN AC REQUERIDA EN INSTALACIÓN } & 9435 & [Wh/día] \\
\hline \multicolumn{5}{|l|}{ MÁXIMO CONSUMO HORA PICO } & 910 & {$[W]$} \\
\hline Equipo & $\begin{array}{l}\text { Potencia } \\
\text { [W] }\end{array}$ & Unidades & $\begin{array}{c}\text { Total potencia } \\
{[\mathrm{W}]}\end{array}$ & Utilización día [h] & $\begin{array}{c}\text { Energía requerida } \\
\text { [Wh/Día] }\end{array}$ & Tipo carga \\
\hline Bombillas LED & 15 & 8 & 120 & 6 & 720 & $\mathrm{AC}$ \\
\hline PC Portátil & 50 & 1 & 50 & 2 & 100 & $\mathrm{AC}$ \\
\hline Router WiFi & 5 & 1 & 5 & 24 & 120 & $A C$ \\
\hline Cámara vigilancia & 5 & 1 & 5 & 24 & 120 & $A C$ \\
\hline Bomba presión & 70 & 1 & 70 & 1 & 70 & DC \\
\hline Teléfono celular & 15 & 1 & 15 & 5 & 75 & $\mathrm{AC}$ \\
\hline Nevera sencilla & 120 & 1 & 120 & 24 & 2880 & $\mathrm{AC}$ \\
\hline Televisor a color & 50 & 1 & 50 & 2 & 100 & $\mathrm{AC}$ \\
\hline Radio - grabadora & 15 & 1 & 15 & 4 & 60 & $\mathrm{AC}$ \\
\hline Pequeños electrodomésticos & 200 & 2 & 15 & 4 & 1600 & $\mathrm{AC}$ \\
\hline Cerca eléctrica & 5 & 1 & 5 & 24 & 120 & $\mathrm{DC}$ \\
\hline Motobomba & 300 & 1 & 300 & 5 & 1500 & $\mathrm{AC}$ \\
\hline Ventiladores & 60 & 3 & 180 & 12 & 2160 & $A C$ \\
\hline
\end{tabular}

Fuente: los autores.

TABLA III

REQUERIMIENTOS DE DISEÑO PARA CONFIGURACIÓN DE CURVA DE CARGA EN CADA HACIENDA

\begin{tabular}{|c|c|c|c|}
\hline Variables de Diseño & Unidad & Variables de Diseño & Unidad \\
\hline Año Base & 2015 & Año de Diseño & 2055 \\
\hline Promedio de habitantes en cada Vivienda & 8 & Factor de Coincidencia de Cargas Horarias & 0,5342 \\
\hline $\mathrm{N}^{\circ}$ Viviendas por Hacienda & 1 & Eficiencia del Sistema de Generación SIN en Colombia & $69 \%$ \\
\hline Tasa Anual de Crecimiento del Consumo & $1,63 \%$ & Pérdidas técnicas del sistema de transmisión y distribución SIN & $14,54 \%$ \\
\hline Potencia Pico (kWp) & 1,1 & Demanda Total de Energía en Cada Hacienda (kWh/año) & 3650 \\
\hline $\begin{array}{l}\text { Coste de Inversión para Transformador } \\
1 \Phi \text { (USD/sistema) }\end{array}$ & 1621 & Coste de Mantenimiento de la Red de Distribución & $3 \%$ \\
\hline Longitud red de distribución (Km) & \multicolumn{3}{|c|}{0,$5 ; 8 ; 15 ; 26 \quad$ (4 haciendas) } \\
\hline
\end{tabular}

Fuente: los autores. 


\subsection{Caracterización del modelo de decisión}

La cantidad de criterios y subcriterios empleados en la planificación de la energía son diversos y numerosos. En consecuencia, una planificación coherente debe incluir criterios de orden económico, técnico, ambiental y social. En principio, la selección de los criterios debería ser realizada directamente por los decisores o agentes de decisión. Sin embargo, poner de acuerdo a los decisores en este proceso de interacción puede resultar una actividad compleja y excesivamente larga. Para evitar esta situación, en virtud de este artículo, se propone un conjunto de criterios y subcriterios elegidos directamente de la literatura y otros que serán aportaciones de este trabajo.

El esquema de la Fig. 3 muestra la estructura jerárquica del problema de decisión. Como vemos, el objetivo global consiste en facilitar la toma de decisiones para escoger el mejor modelo de suministro eléctrico y la configuración de tecnologías acorde con los criterios y subcriterios de los siguientes niveles; se ubica en el tope o máximo nivel de jerarquía (nivel 1).

Al respecto, y para este fin de realización del caso de aplicación, se han elegido 13 subcriterios, entre los cuales se encuentran los siguientes: coeficiente de eficiencia, energía no servida, seguridad en la disponibilidad del recurso energético, fiabilidad de la tecnología, coste actual neto, coste variable de operación y mantenimiento, calentamiento global, acidificación, eutrofización, uso de la tierra, aceptación social de la energía, creación de empleo y el Índice de Desarrollo Humano.

Con el criterio técnico, compuesto por el coeficiente de eficiencia (ECF), energía no servida (ENS), disponibilidad del recurso energético a largo plazo y fiabilidad de la tecnología (FT), se pretende evaluar la factibilidad o funcionabilidad del sistema de suministro de electricidad; teniendo en cuenta diferenciar el sistema que mejor aprovechamiento de los recursos energéticos locales, con unos recursos que estén garantizados a largo plazo, disponibles en gran parte del año para satisfacer la demanda y con unas tecnologías de conversión de una fiabilidad aceptable.

A través del criterio económico, subdivido en el coste actual neto (CAN) y coste variable de operación y mantenimiento de las tecnologías (CVOM), se pretende evaluar la viabilidad del proyecto. Para ello, un sistema será viable económicamente cuando aporte el menor coste total al inicio de la inversión (coste de inversión, coste de operación y mantenimiento fijo, combustible, etc.) y el menor coste variable de operación y mantenimiento. La viabilidad económica garantiza que el sistema sea asequible por los futuros usuarios de la energía y también por parte de los inversores, si este corresponde a la administración pública.

Fig. 3. ESTRUCTURA JERÁRQUICA PARA LA SELECCIÓN DEL MODELO Y TECNOLOGÍA DE SUMINISTRO ELÉCTRICO

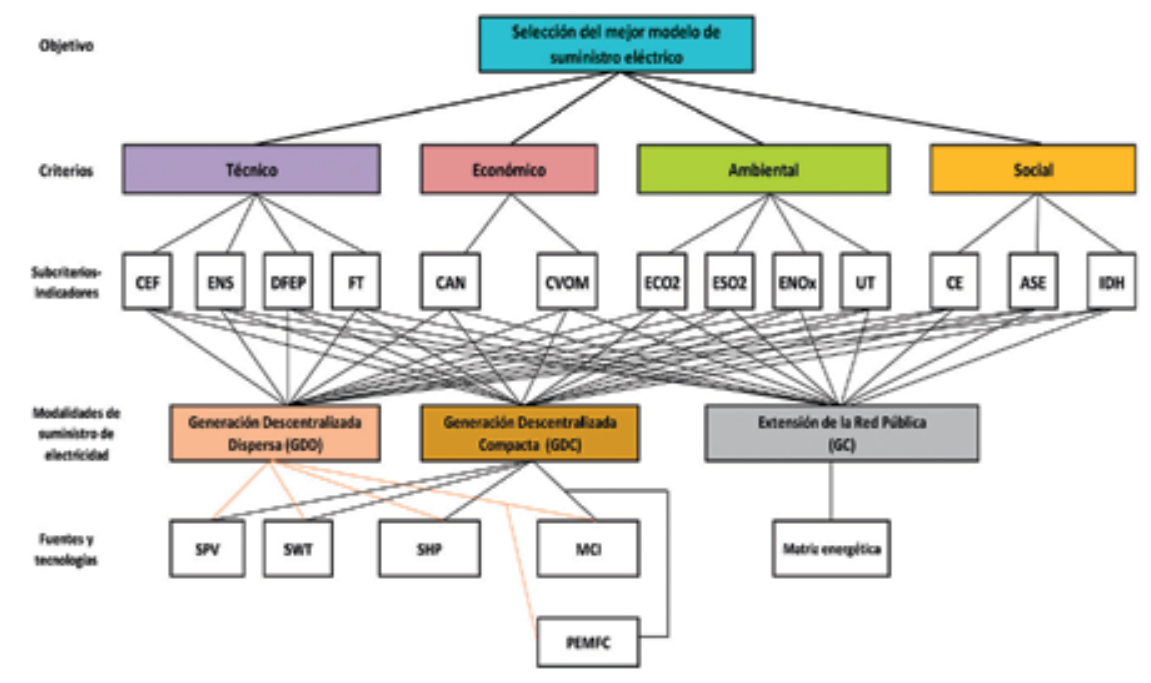

Fuente: [19] 
Desde el punto de vista ambiental, con los subcriterios propuestos, tales como calentamiento global, acidificación, eutrofización y uso de la tierra, se pretende evaluar el sistema de suministro eléctrico que implique el menor impacto ambiental. En esta evaluación se han elegido los subcriterios de acuerdo con su importancia desde el punto de vista global, como el cambio climático (emisiones de $\mathrm{CO}_{2}$ ), regional, como la acidificación o lluvia ácida (emisiones de $\mathrm{SO}_{2}$ ) y su impacto local, asociado a la eutrofización (emisiones de $\mathrm{NO}_{x}$ ) y el uso de la tierra. Para propiciar una evaluación lo más objetivamente posible se utiliza el análisis de ciclo de vida (ACV) y así valorar las emisiones de todas las tecnologías.

Finalmente, mediante el criterio social, subdividido en tres subcriterios, tales como aceptación social de la energía (ASE), creación de empleo (CE) y el índice de desarrollo humano (IDH), se pretende realizar una evaluación que permita diferenciar la alternativa que mayor desarrollo social aporte a la comunidad. Es importante acotar en relación con la aceptación social de la energía, el nivel de participación que tienen los potenciales usuarios del futuro sistema de suministro eléctrico en valorarlo directamente. En referencia al IDH, este elemento aporta información sobre el nivel de desarrollo socioeconómico, educación y esperanza de vida; siendo esto un aspecto de amplio interés en el ámbito local y desafortunadamente muy poco explorado hasta el momento.

\subsection{Construcción del Modelo de Energización en ZNI}

Las cargas del sistema pueden ser: cargas eléctricas en corriente alterna ( $A C)$, cargas eléctricas en corriente continua (DC). La combinación de fuentes y tecnologías, así como las modalidades de suministro de electricidad que se contemplan en la metodología resumida en la Fig. 3 , se procesan a través de un modelo de toma de decisiones optimizado, el cual se ha implementado computacionalmente en el software i-HOGA [12], [34], [35], el cual evalúa todas las posibles combinaciones, tanto de componentes como de variables de control de la estrategia, es decir, optimización, ya sea por medio de un método enumerativo o "fuerza bruta", o a través de algoritmos genéticos. Este atributo hace que HOGA sea más competitivo que HOMER, software desarrollado por National Renewable Energy Laboratory [36], [37].

i-HOGA, al usar algoritmos genéticos, permite encontrar soluciones muy cercanas a la óptima con tiempos de cálculo muy bajos. Adicionalmente, i-HOGA toma en cuenta los costes de reposición, la inflación esperada y el límite de modificación de precios respecto a los actuales. Este último aspecto es bastante interesante para considerar una inflación negativa en los equipos, lo cual permitiría incluir la tasa de reducción de costes en función de su madurez tecnológica.

La Tabla IV proporciona información básica sobre las tecnologías vigentes en el mercado de generación distribuida, que permiten obtener las configuraciones óptimas en términos económicos, técnicos y ambientales, con la cual se estableció el modelo de simulación i-HOGA, teniendo en cuenta las coordenadas geográficas (Latitud $=$ $+3,17^{\circ}$, Longitud $=-73^{\circ}$ ).

El modelo de simulación también tiene en cuenta la existencia de inversores monofásicos, cuyas potencias oscilan entre 0.45 y $3.6 \mathrm{~kW}$, así como reguladores para protección de los bancos de batería[38].

La Fig. 4 proporciona los resultados de soluciones no dominadas, entregadas por i-HOGA, las cuales se ubican en la frontera óptima de $\mathrm{Pa}$ reto, teniendo en cuenta los requerimientos de inversiones económicas, medida por el valor actual neto (en euros) y el desempeño técnico de la solución energética, medida como energía no suministrada respecto de la curva de carga para cada hacienda ( $\mathrm{hWh} / \mathrm{año}$ ). En total, se estiman 38 posibles combinaciones o alternativas de configuración de tecnologías de generación distribuida para cada una de las viviendas que requieren ser energizadas.

La elección de la mejor alternativa de energización tendrá en cuenta la ponderación de criterios y subcriterios considerados en la planificación energética, desde el punto de vista técnico, económico, ambiental y social. Asimismo, se tiene en cuenta la comparación con el sistema de generación centralizado, en el que los costos de extensión de una red de distribución de medio voltaje se estima en 15.000 dólares americanos por Kilómetro [39]. 
TABLA IV

PARÁMETROS DE CONFIGURACIÓN DEL MODELO ENERGÉTICO EN i-HOGA

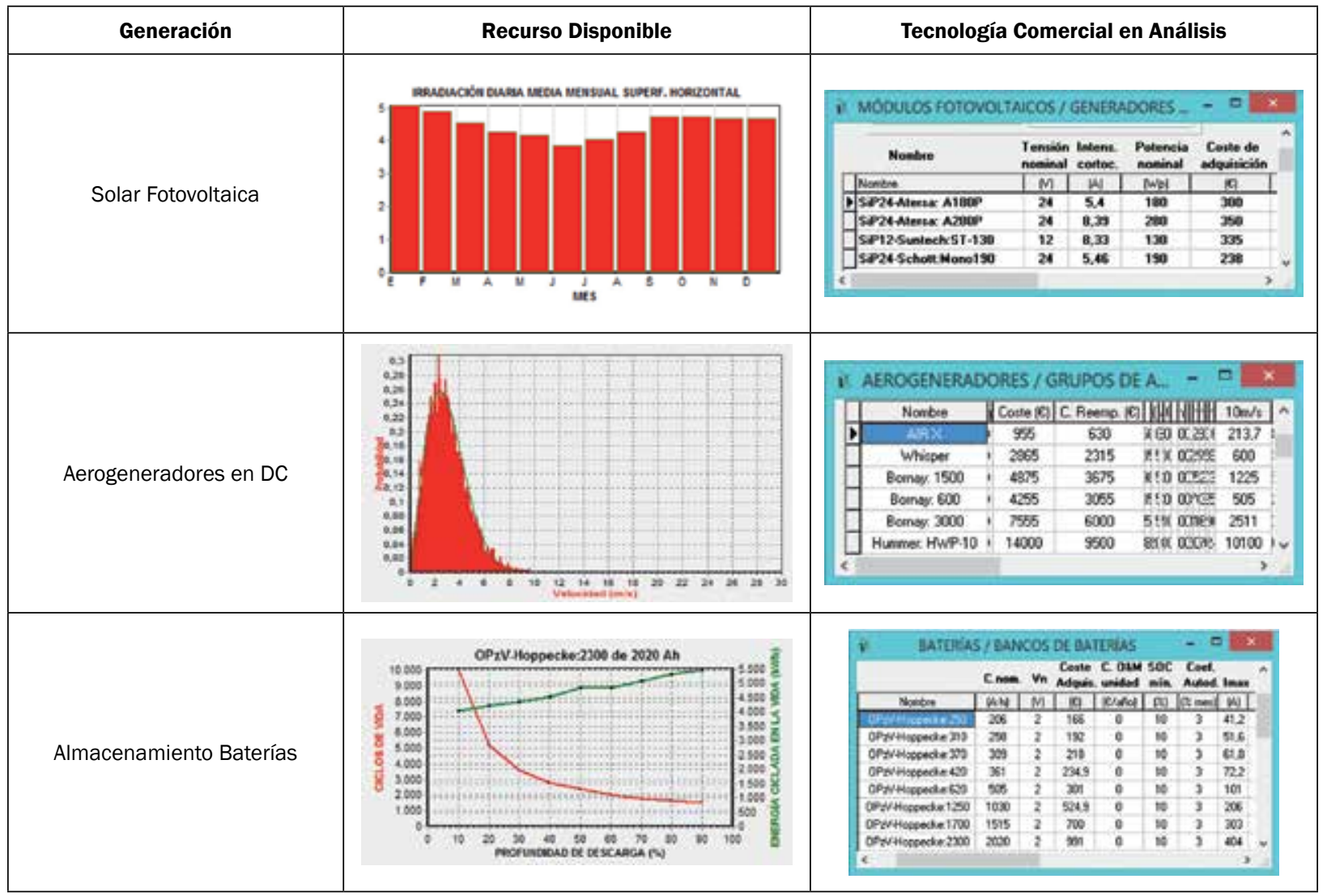

Fuente: los autores.

Fig. 4. SOLUCIONES NO DOMINADAS Y CONFIGURACIÓN ÓPTIMA DEL SISTEMA DE GD

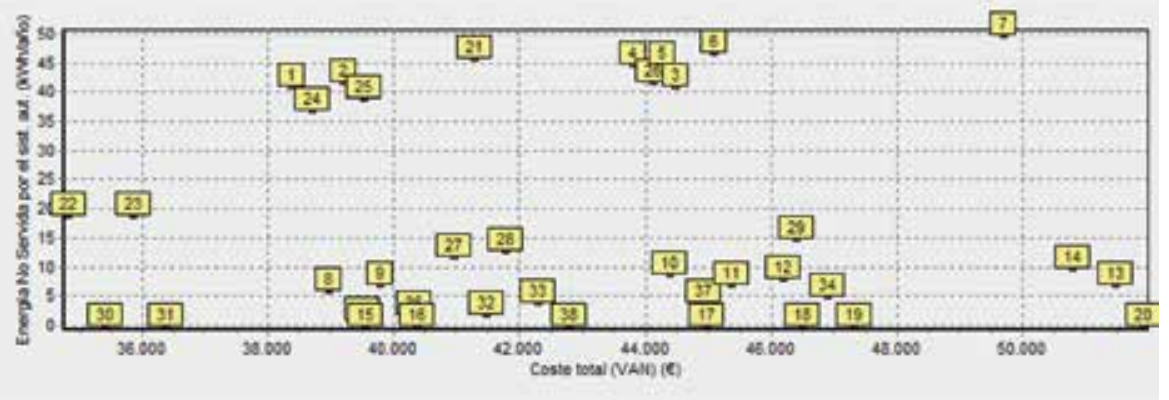

Fuente: los autores.

\subsection{Construcción de la matriz de decisión y criterios de ponderación}

Una matriz de decisión resume la evaluación de cada alternativa conforme a los criterios y subcriterios (en función de su correspondiente indicador) de la estructura jerárquica. La escala de medida de las evaluaciones puede ser cuantitativa o cualitativa, y las medidas pueden expresarse en escala cardinal, ordinal, nominal y probabilística.
Para la construcción de la matriz de decisión, se tienen en cuenta los factores, como: opciones de decisión, listado de soluciones no dominadas de i-HOGA, indicadores y criterios requeridos para la planificación energética. Una opción de construcción de dicha matriz de decisión se presenta en [26]also in energy planning. However, social and environmental criteria have not been fully integrated in rural electrification projects design and play often an opposing role to technical and economic 
criteria. We propose the combined application of two multi-criteria decision-making methods, namely, the Analytical Hierarchy Process (AHP. Para el caso del estudio en esta contribución técnica se obtienen los resultados que se muestran en la Tabla V. De las 38 posibles soluciones óptimas, se ha realizado un filtro de las 14 alternativas más económicas. Asimismo, se presenta la comparación con la posible alternativa de extensión de la red de distribución de medio voltaje.

Dadas las características del problema de decisión (problema de decisión multicriterio complejo, de tipo discreto, bajo certidumbre y donde varios expertos intervienen en el proceso de decisión a través de un conjunto de criterios representativos), le son aplicables algunos métodos de decisión multicriterio como el Proceso Analítico Jerárquico (AHP), que por sus particularidades descritas en la sección 3.1 se aplica aquí a la metodología propuesta. Para el efecto, se ha conformado un grupo de expertos para la ponderación de los criterios que aparecen en la Tabla V, con la estimación de preferencias individuales en una sola valoración colectiva.
Adicionalmente, el ajuste del esquema de decisiones con metodología AHP se complementa con la aplicación de VIKOR debido a la existencia de criterios en conflicto, no cuantificables. Para la respuesta final, se presenta un ordenamiento de soluciones compromiso, fundamentado en la comparación con la solución ideal, es decir, en la medida de proximidad a la solución ideal.

La Tabla VI proporciona la ponderación de pesos obtenida a partir de entrevistas cerradas dirigidas a 11 científicos expertos, cuyo grupo estuvo integrado por académicos, representantes de empresas y consultoras, reguladores o entes de la administración pública y organismos no gubernamentales (ONG), quienes participaron con la calificación de criterios bajo metodología AHP [19]. La toma de decisiones multicriterio en el problema de planificación implica la participación de expertos o decisores en diferentes momentos del proceso de análisis de decisión. Al respecto, en esta contribución técnica la intervención de los expertos se limita a la estimación de preferencias o ponderación de los criterios, subcriterios y categoría del grupo de expertos.

TABLA V

MATRIZ DE DECISIÓN PARA SELECCIÓN DE LA MEJOR CONFIGURACIÓN DE GD PARA VIVIENDA MÁS AISLADA

\begin{tabular}{|l|c|c|c|c|c|c|c|c|c|}
\hline $\begin{array}{c}\text { Alternativas } \\
\text { GDD }\end{array}$ & Costes VAN (€) & $\begin{array}{c}\text { Costo } \\
\text { O\&M } \\
(€ / \text { año })\end{array}$ & $\begin{array}{c}\text { Emisión } \\
\mathrm{CO}_{2} \\
(\mathrm{~kg} / \mathrm{año}) \\
\text { Alternativa 1 }\end{array}$ & $\begin{array}{c}\text { Emisión } \\
\mathrm{SO}_{\mathrm{x}} \\
(\mathrm{kg} / \mathrm{año}) \\
0,89\end{array}$ & $\begin{array}{c}\text { Emisión } \\
\mathrm{NO}_{\mathrm{x}} \\
(\mathrm{kg} / \mathrm{año}) \\
0,48\end{array}$ & $\begin{array}{c}\text { Uso de } \\
\text { suelo }\left(\mathrm{m}^{2}\right)\end{array}$ & $\begin{array}{c}\text { Aceptación } \\
\text { social (\%) }\end{array}$ & $\begin{array}{c}\text { Indice } \\
\text { Desarrollo } \\
\text { Humano }\end{array}$ & $\begin{array}{c}\text { ENS } \\
\text { (kWh/ } \\
\text { año) }\end{array}$ \\
\hline Alternativa 2 & $39.202,29$ & 35,72 & 234,99 & 0,89 & 0,48 & 209 & $76,18 \%$ & 0,6336 & 42,55 \\
\hline Alternativa 3 & $38.965,29$ & 38,30 & 247,48 & 0,96 & 0,52 & 218 & $75,91 \%$ & 0,6346 & 5,71 \\
\hline Alternativa 4 & $39.786,79$ & 38,30 & 243,95 & 0,96 & 0,52 & 218 & $75,91 \%$ & 0,6345 & 6,84 \\
\hline Alternativa 5 & $39.549,80$ & 40,88 & 256,44 & 1,03 & 0,56 & 227 & $75,67 \%$ & 0,6347 & 0,00 \\
\hline Alternativa 6 & $40.371,30$ & 40,88 & 252,91 & 1,03 & 0,56 & 227 & $75,67 \%$ & 0,6347 & 0,00 \\
\hline Alternativa 7 & $34.835,75$ & 50,32 & 337,35 & 1,20 & 0,66 & 881 & $71,70 \%$ & 0,6342 & 18,72 \\
\hline Alternativa 8 & $35.858,30$ & 50,32 & 335,19 & 1,20 & 0,66 & 881 & $71,70 \%$ & 0,6342 & 19,29 \\
\hline Alternativa 9 & $38.708,10$ & 41,86 & 243,32 & 0,89 & 0,48 & 923 & $76,16 \%$ & 0,6337 & 37,18 \\
\hline Alternativa 10 & $39.529,61$ & 41,86 & 239,79 & 0,89 & 0,48 & 923 & $76,16 \%$ & 0,6337 & 38,93 \\
\hline Alternativa 11 & $35.414,17$ & 52,90 & 345,84 & 1,27 & 0,70 & 890 & $71,68 \%$ & 0,6347 & 0,00 \\
\hline Alternativa 12 & $36.373,43$ & 52,90 & 343,69 & 1,27 & 0,70 & 890 & $71,68 \%$ & 0,6347 & 0,00 \\
\hline Alternativa 13 & $39.503,04$ & 297,73 & 255,48 & 14,75 & 9,05 & 2.684 & $57,14 \%$ & 0,6347 & 1,20 \\
\hline Alternativa 14 & $40.324,54$ & 39,24 & 251,95 & 10,27 & 0,54 & 135.952 & $0,00 \%$ & 0,6347 & 1,77 \\
\hline Alternativa Red & $394.849,73$ & 18,73 & $2.962,45$ & 0,84 & 2,96 & $1 \times 106$ & $0,00 \%$ & 0,6336 & 0,00 \\
\hline Eléctrica & & & & & & & \\
\hline
\end{tabular}

Fuente: los autores. 
TABLA VI

PONDERACIÓN DE CRITERIOS DE DECISIÓN, MEDIANTE METODOLOGÍA AHP

\begin{tabular}{|c|c|c|}
\hline Importancia criterio & \multicolumn{2}{|l|}{ Importancia relativa subcriterios } \\
\hline \multirow{2}{*}{ Económico $(20,72 \%)$} & Coste actual neto & $16,58 \%$ \\
\hline & Coste variable de operación y mantenimiento & $4,14 \%$ \\
\hline \multirow{4}{*}{ Ambiental $(22,48 \%)$} & Emisiones de $\mathrm{CO}_{2}$ & $6,34 \%$ \\
\hline & Emisiones de $\mathrm{SO}_{2}$ & $3,50 \%$ \\
\hline & Emisiones de $\mathrm{NO}_{\mathrm{x}}$ & $5,26 \%$ \\
\hline & Uso de la tierra & $7,38 \%$ \\
\hline \multirow{3}{*}{ Social $(26,56 \%)$} & Aceptación social de la energía & $8,87 \%$ \\
\hline & Creación de empleo & $7,55 \%$ \\
\hline & Índice de desarrollo humano & $10,23 \%$ \\
\hline \multirow{4}{*}{ Técnico $(30,14 \%)$} & Coeficiente de eficiencia & $7,05 \%$ \\
\hline & Disponibilidad a largo plazo del recurso energético & $6,81 \%$ \\
\hline & Fiabilidad de las tecnologías & $10,29 \%$ \\
\hline & Energía no servida & $6,00 \%$ \\
\hline
\end{tabular}

Fuente: los autores.

Desde el punto de vista multicriterio, la elección de la mejor alternativa requiere la incorporación de otra técnica de apoyo a la toma de decisiones que permita ordenarlas objetivamente desde la mejor hasta la peor (ranking de clasificación). Para ello, a continuación se aplica el método MCDM-VIKOR comprensivo en la siguiente fase del proceso de análisis o toma de decisiones, que tiene en cuenta la normalización de las alternativas resumidas en la matriz de decisión de la Tabla $\mathrm{V}$, a la cual también se le aplica la normalización descrita previamente en la ecuación (2).

Seguidamente, se han implementado las ecuaciones (4), (5) y (6) para la obtención de las métricas $S_{i}$ (medida de utilidad o total satisfacción), $R_{i}$ (medida de pesar o insatisfacción) y $Q_{i}$ que corres- ponde al valor de clasificación de VIKOR para la $i$ ésima alternativa, según se aprecia en la Tabla VII.

\subsection{Resultado del ejercicio de planificación}

A partir de la combinación de las métricas y coeficientes de ponderación presentada previamente, se obtienen tres soluciones ordenadas de clasificación (ranking), en las que la alternativa que tenga el menor valor de Qi representa temporalmente la opción más favorable. Finalmente, se han aplicado las ecuaciones (7) y (9) en la verificación de la estabilidad de la clasificación de soluciones compromiso, con lo que es posible construir la siguiente Tabla VIII resumen, que contiene la mejor configuración del conjunto de soluciones óptimas presentadas en la Fig. 4.

TABLA VII

MÉTRICAS DE COEFICIENTES DE DISTANCIA METODOLOGÍA VIKOR (Si, Ri, Qi), RESPECTO A SOLUCIÓN IDEAL EN PROBLEMA DE PLANIFICACIÓN

\begin{tabular}{|l|c|c|c|c|}
\hline Coeficientes VIKOR & Hacienda 1 & Hacienda 2 & Hacienda 3 & Hacienda 4 \\
\hline Distancia métrica Si & 0,16388032 & 0,06555318 & 0,0619805 & 0,05983682 \\
\hline Distancia métrica Ri & 0,06501765 & 0,03323839 & 0,03323839 & 0,03323839 \\
\hline Distancia Qi=0,5 & 0,21936703 & 0,01054022 & 0,00746106 & 0,0064837 \\
\hline Distancia Qi=0,25 & 0,25 & 0,01175381 & 0,01021423 & 0,00972555 \\
\hline Distancia Qi=0,75 & 0,10968352 & 0,00932662 & 0,00470789 & 0,00324185 \\
\hline
\end{tabular}

Fuente: los autores. 
La Tabla VIII presenta el resultado de aplicar los coeficientes de ponderación derivados de la metodología AHP, y el resultado de aplicar las métricas de distancia respecto a la solución ideal del método VIKOR, sobre la matriz normalizada. El ordenamiento de alternativas previamente abordadas en la Tabla $V$ proporciona la mejor solución al problema de planificación óptima utilizando tecnología de Generación Distribuida, en este caso, aplicada a la Hacienda 4, que es la que se encuentra más aislada geográficamente (a $26 \mathrm{Km}$ de la carretera principal).

En este caso, llama la atención el hecho de que la alternativa de extender la Red de Distribución hasta la Hacienda 4, a una distancia de 26 $\mathrm{km}$, constituye la opción menos efectiva, y tampoco constituye una solución comprometida para el problema de energización rural en la zona no interconectada.
La Tabla IX proporciona información definitiva sobre el ordenamiento de las mejores soluciones para el conjunto de viviendas en las 4 haciendas en estudio. Dichas alternativas tienen la conveniencia de garantizar un suministro constante de energía durante el tiempo de funcionamiento del sistemas (ENS $=0$ ), así como las menores inversiones económicas en el periodo de planificación de 40 años.

Esta modalidad implica la generación del recurso eléctrico en las adyacencias de la propia vivienda o edificación. Para ello, la oferta compuesta por una o varias tecnologías de generación, incluido el almacenamiento (baterías), debe satisfacer los requerimientos de energía de las viviendas y de los elementos de cada hacienda. En términos prácticos, un ejemplo de GD lo representa un sistema solar doméstico, cuyo esquema más detallado de esta modalidad se muestra en la Fig.5.

TABLA VIII

ORDENAMIENTO DE SOLUCIONES ÓPTIMAS Y COMPROMETIDAS PARA PLANIFICACIÓN DE GD EN LA HACIENDA 4

\begin{tabular}{|c|c|c|c|c|c|}
\hline $\begin{array}{l}\text { ORDEN } \\
1\end{array}$ & $\begin{array}{c}\text { Métrica Si } \\
0,0598\end{array}$ & $\begin{array}{c}\text { Métrica Ri } \\
0,0332\end{array}$ & $\begin{array}{c}\text { Métrica Qi=0,5 } \\
0,0065\end{array}$ & $\begin{array}{l}\text { Correspondencia } \\
\text { Alternativa } 5\end{array}$ & $\begin{array}{l}\text { Observación } \\
\text { Solución compromiso }\end{array}$ \\
\hline 2 & 0,0599 & 0,0339 & 0,0068 & Alternativa 6 & Solución compromiso \\
\hline 3 & 0,0601 & 0,0339 & 0,0325 & Alternativa 3 & Solución compromiso \\
\hline 4 & 0,0605 & 0,0342 & 0,0375 & Alternativa 4 & Solución compromiso \\
\hline 5 & 0,0823 & 0,0342 & 0,0431 & Alternativa 11 & Solución compromiso \\
\hline 6 & 0,0863 & 0,0394 & 0,0438 & Alternativa 12 & Solución compromiso \\
\hline 7 & 0,1216 & 0,0394 & 0,1028 & Alternativa 13 & No es solución del problema \\
\hline 8 & 0,1234 & 0,0394 & 0,1199 & Alternativa 7 & No es solución del problema \\
\hline 9 & 0,1423 & 0,0394 & 0,1221 & Alternativa 8 & No es solución del problema \\
\hline 10 & 0,1638 & 0,0560 & 0,3172 & Alternativa 9 & No es solución del problema \\
\hline 11 & 0,1666 & 0,0596 & 0,3330 & Alternativa 10 & No es solución del problema \\
\hline 12 & 0,1666 & 0,0613 & 0,3383 & Alternativa 1 & No es solución del problema \\
\hline 13 & 0,1696 & 0,0631 & 0,3528 & Alternativa 2 & No es solución del problema \\
\hline 14 & 0,2295 & 0,0647 & 0,3708 & Alternativa 14 & No es solución del problema \\
\hline 15 & 0,4611 & 0,1048 & 1,0000 & Alternativa Red Eléctrica & No es solución del problema \\
\hline
\end{tabular}

Fuente: los autores.

TABLA IX

SOLUCIONES ÓPTIMAS Y COMPROMETIDAS PARA PLANIFICACIÓN DE GD EN LAS 4 HACIENDAS

\begin{tabular}{|l|c|c|c|c|}
\hline & Hacienda 1 & Hacienda 2 & Hacienda 3 & Hacienda 4 \\
\hline Métrica Qi=0,5 & 0,21936703 & 0,01054022 & 0,00746106 & 0,0064837 \\
\hline Correspondencia & Alternativa 11 & Alternativa 5 & Alternativa 5 & Alternativa 5 \\
\hline
\end{tabular}

Fuente: Ios autores. 
Fig. 5. ESQUEMA GENERAL DE LA GENERACIÓN DISTRIBUIDA

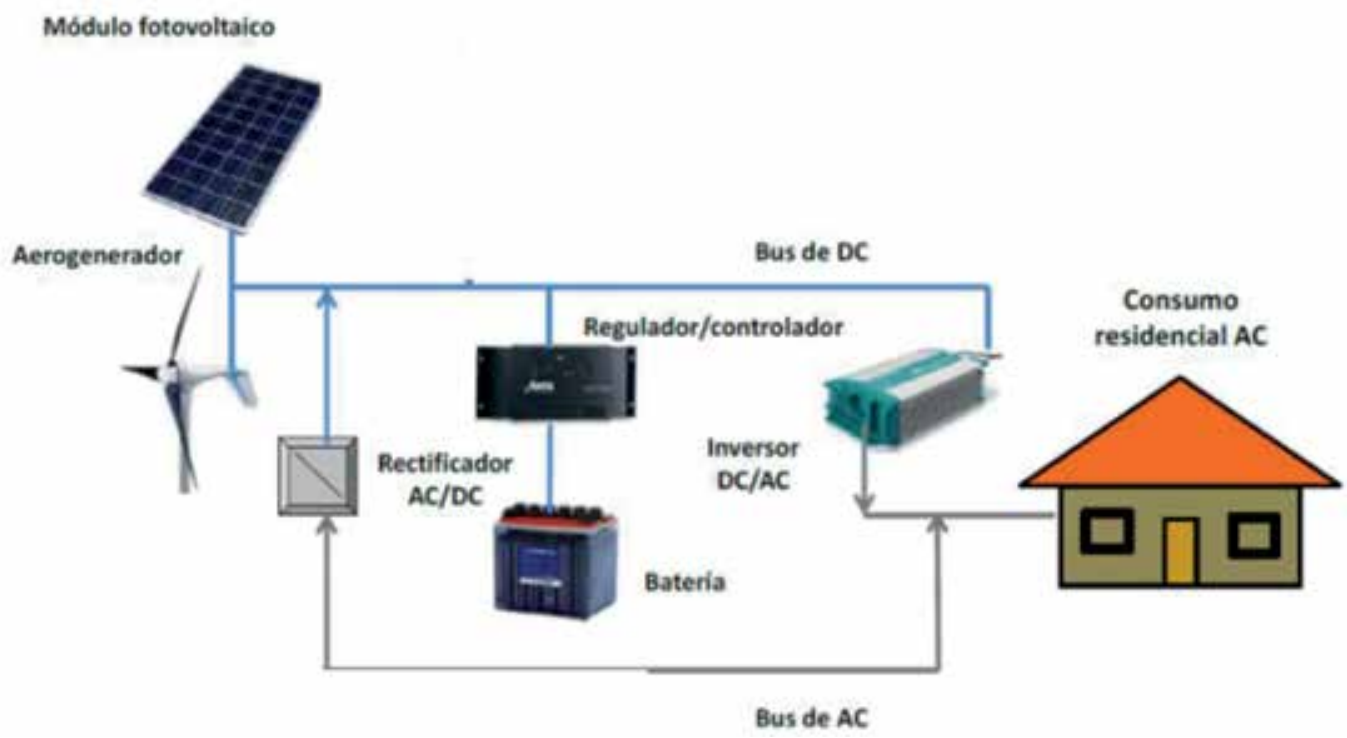

Fuente: los autores.

La configuración tecnológica de la Alternativa 11, que corresponde a la mejor decisión para energizar la Hacienda 1 se toma directamente de la aplicación i-HOGA. La Alternativa 5, por su parte, corresponde a la mejor configuración para energización de las Haciendas 2, 3 y 4, identificadas en el mapa de la Fig. 1. En la Tabla X se proporciona el respectivo esquema, así como el diseño de la configuración tecnológica para asegurar la energización de las viviendas en las haciendas.

En resumen, la solución de planificación energética para la ZNI, tiene en cuenta el aprovechamiento del potencial eólico y solar de las haciendas que requieren el suministro energético. Para los efectos de este artículo, vale la pena aclarar que dicha información se puede acceder fácilmente a través de los atlas de radiación solar y el mapa de vientos disponibles para el punto geográfico[40], [41]. Para el caso de la Hacienda 1, que está a orillas de la carretera Puerto Concordia - Puerto Lleras, la solución más eficiente desde el punto de vista ambiental, técnico, económico y social, consiste en el montaje de un grupo fotovoltaico de 5040Wp (aproximadamente 18 páneles FV de $280 \mathrm{Wp}$ cada uno) con un pequeño aerogenerador de 545W (en este caso, puede ser el aerogenerador Whisper). En tanto que para las demás haciendas, ubicadas en territorios aislados dentro de la vereda del municipio de Puerto Lleras, se contempla la instalación de un aeroge- nerador de 1660W (en este caso, puede ser el aerogenerador Bornay 1500) y un grupo fotovoltaico de $3640 W p$ (aproximadamente 13 páneles FV de 280 Wp cada uno).

\section{CONCLUSIONES}

Se ha proporcionado un marco metodológico para la aplicación de métodos multicriterio AHP y VIKOR a la planificación o diseño de sistemas de suministro eléctrico en áreas rurales o remotas de países en vías de desarrollo, cuya efectividad ha quedado evidenciada en el estudio de un caso real en el municipio de Puerto Lleras (vereda las Gaviotas), para lo cual se han utilizado alternativas óptimas multiobjetivo para la generación descentralizada, y múltiples criterios de orden técnico, económico, ambiental y social en la toma de decisiones. De esta manera, se ha abordado el problema de planificación de la generación distribuida para áreas rurales, con una visión holística lo que implica la integración de criterios ambientales y sociales, además de los convencionales (técnicos y económicos), en la toma de decisiones, conduciendo al tratamiento de un problema multidimensional.

El criterio ambiental toma en cuenta el ámbito de aplicación del impacto, contextualizando de esta manera los efectos que se producen a nivel global (calentamiento global), regional (Iluvia ácida) y local (eutrofización y uso del territorio). 
TABLA X

ESQUEMA DE SOLUCIÓN PARA IMPLEMENTACIÓN DE LA ENERGIZACIÓN EN LAS HACIENDAS

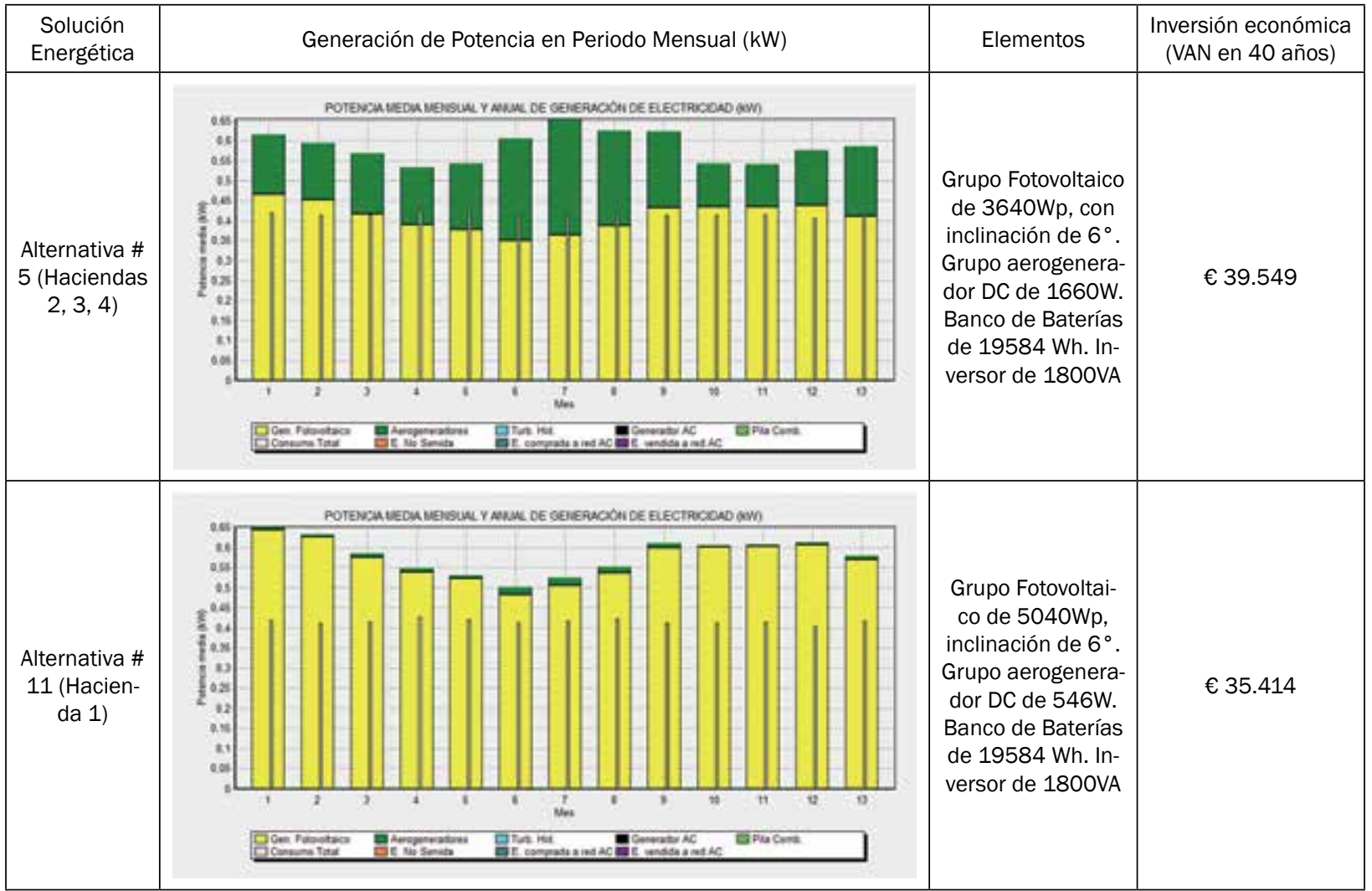

Fuente: los autores.

La propuesta metodológica es de gran utilidad para la planificación que los gobiernos y las empresas de servicios públicos realizan respecto de sus consumidores ubicados en las zonas no interconectadas. Se ha evidenciado que en la gran mayoría de los casos, las mejores soluciones no deben limitarse a extender las líneas de distribución de media tensión. Por el contrario, es evidente la penetración de las tecnologías de generación distribuida, con precios cada vez más competitivos y con mejor desempeño técnico, además de la posibilidad de extensión de su vida útil a partir de buenas prácticas de operación y mantenimiento por parte de sus usuarios.

\section{REFERENCIAS}

[1] World_Bank, Designing Sustainable Off-Grid Rural Electrification Projects: Principles and Practices, Operationa. Washington DC (EEUU): World Bank, 2008.

[2] Fenercom, "Boletín Energético N5", en I Congreso de Generación Distribuida, 2009, p. 12p.
[3] G. J. Correa-Henao y J. M. Yusta-Loyo, Seguridad en Infraestructuras de Transporte de Electricidad, 1.a ed. Saarbrücken, Alemania: Editorial Académica Española, 2014.

[4] G. J. Correa-Henao y J. M. Yusta-Loyo, "Seguridad Energética y Protección de Infraestructuras Críticas", Lámpsakos, vol. 1, n.o 10, pp. 92-108, 2013.

[5] T. Saaty, The analytic hierarchy process: planning, priority setting, resource allocation. New York, NY (USA): McGraw-Hill International Book Co., 1980.

[6] G.-H. Tzeng, M.-H. Teng, J.-J. Chen, y S. Opricovic, “Multicriteria selection for a restaurant location in Taipei", Int. J. Hosp. Manag., vol. 21, n.o 2, pp. 171-187, jun. 2002.

[7] S. Opricović, "Dynamic compromise programming with application to water reservoir management", Agric. Syst., vol. 41, n.o 3, pp. 335-347, 1993.

[8] G.-H. Tzeng, C.-W. Lin, y S. Opricovic, "Multi-criteria analysis of alternative-fuel buses for public transportation", Energy Policy, vol. 33, n.o 11, pp. 1373-1383, jul. 2005. 
[9] P. L. Yu y M. Zeleny, "The set of all nondominated solutions in linear cases and a multicriteria simplex method", J. Math. Anal. Appl., vol. 49, n.o 2, pp. 430468, feb. 1975.

[10] G. Colson y M. Zeleny, "Multicriterion concept of risk under incomplete information", Comput. Oper. Res., vol. 7, n.o 1-2, pp. 125-141, 1980.

[11] M. Zeleny, "The pros and cons of goal programming", Comput. Oper. Res., vol. 8, n.o 4, pp. 357-359, 1981.

[12] R. Dufo, "iHOGA: Sistemas Híbridos Renovables", 2015. [En línea]. Disponible en: http://bit.ly/1I2WBsr.

[13] J. M. Mantilla-González, C. A. Duque-Daza, y C. H. Galeano-Urueña, "Análisis del esquema de generación distribuida como una opción para el sistema eléctrico colombiano", Rev. Fac. Ing. Univ. Antioquia, n.o 44, pp. 97-110, 2008.

[14] Á. A. Bayod-Rújula, Sistemas fotovoltaicos. Zaragoza, España: Universidad de Zaragoza, 2009.

[15] ElA, Distributed generation in liberalized electricity markets. OECD/IEA. Paris, Francia, 2002.

[16] O. Pol, P. Palensky, C. Kuh, K. Leutgöb, J. Page, y G. Zucker, "Integration of centralized energy monitoring specifications into the planning process of a new urban development area: a step towards smart cities", e i Elektrotechnik und Informationstechnik, vol. 129, n.o 4, pp. 258-264, 2012.

[17] K. Alanne y A. Saari, "Distributed energy generation and sustainable development", Renew. Sustain. Energy Rev., vol. 10, n.o 6, pp. 539-558, dic. 2006.

[18] J. C. Rojas-Zerpa y J. M. Yusta-Loyo, "Methodologies, technologies and applications for electric supply planning in rural remote areas", Energy Sustain. Dev., vol. 20, n.o 0, pp. 66-76, 2014

[19] J. C. Rojas-Zerpa, "Planificación del suministro eléctrico en áreas rurales de los países en vías de desarrollo: un marco de referencia para la toma de decisiones", Universidad de Zaragoza, 2013.

[20] Á. A. Bayod-Rújula y N. Dia-Khalidou, "Application of a multi-criteria analysis for the selection of the most suitable energy source and water desalination system in Mauritania”, Energy Policy, vol. 38, n.o 1, pp. 99-115, 2010.

[21] T. Ackermann, G. Andersson, y L. Söder, "Overview of government and market driven programs for the promotion of renewable power generation", Renew. Energy, vol. 22, n.o 1-3, pp. 197-204, ene. 2001.

[22] G. Pepermans, J. Driesen, D. Haeseldonckx, R. Belmans, y W. D'haeseleer, “Distributed generation: definition, benefits and issues", Energy Policy, vol. 33, n.o 6, pp. 787-798, abr. 2005
[23] G. J. Correa-Henao, Metodologías para la toma de decisiones apoyadas en modelos difusos. Saarbrücken, Alemania: Editorial Académica Española, 2015.

[24] R. B. Hiremath, S. Shikha, y N. H. Ravindranath, “Decentralized energy planning; modeling and application-a review", Renew. Sustain. Energy Rev., vol. 11, n.o 5, pp. 729-752, jun. 2007.

[25] F. Henao, J. A. Cherni, P. Jaramillo, y I. Dyner, "A multicriteria approach to sustainable energy supply for the rural poor", Eur. J. Oper. Res., vol. 218, n.o 3, pp. 801809, may 2012.

[26] J. C. Rojas-Zerpa y J. M. Yusta, "Application of multicriteria decision methods for electric supply planning in rural and remote areas", Renew. Sustain. Energy Rev., vol. 52, pp. 557-571, dic. 2015.

[27] T. L. Saaty, "Highlights and critical points in the theory and application of the Analytic Hierarchy Process", Eur. J. Oper. Res., vol. 74, n.o 3, pp. 426-447, 1994.

[28] Expert_Choice_Team, "Expertchoice for Collaborative Decision Making", Expert Choice Case Studies, 2014. [En línea]. Disponible en: http://expertchoice.com/. [Accedido: 01-nov-2015].

[29] G.-N. Zhu, J. Hu, J. Qi, C.-C. Gu, y Y.-H. Peng, "An integrated AHP and VIKOR for design concept evaluation based on rough number", Adv. Eng. Informatics, vol. 29, n.o 3, pp. 408-418, ago. 2015.

[30] Y. Zhou, K. Maumbe, J. Deng, y S. W. Selin, “Resourcebased destination competitiveness evaluation using a hybrid analytic hierarchy process (AHP): The case study of West Virginia", Tour. Manag. Perspect., vol. 15, pp. 72-80, jul. 2015.

[31] A. Jahan, F. Mustapha, M. Y. Ismail, S. M. Sapuan, y M. Bahraminasab, "A comprehensive VIKOR method for material selection", Mater. Des., vol. 32, n.o 3, pp. 1215-1221, mar. 2011

[32] N. K. Dia, Á. A. Bayod-Rújula, N. Mamoudou, C. S. Ethmane, y B. O. Bilal, "Field study of multifunctional platforms in Mauritania", Energy Sustain. Dev., vol. 23, n.o 0, pp. 130-140, 2014.

[33] OECD/IEA, "Energy poverty: How to make modern energy access universal?", Paris, Francia, 2010.

[34] J. A. Domínguez-Navarro, J. L. Bernal-Agustín, y R. Dufo-López, "Data mining methodology for disaggregation of load demand", Electr. Power Syst. Res., vol. 79, n.o 10, pp. 1393-1399, oct. 2009.

[35] J. L. Bernal-Agustín y R. Dufo-López, "Simulation and optimization of stand-alone hybrid renewable energy systems", Renew. Sustain. Energy Rev., vol. 13, n.o 8, pp. 2111-2118, oct. 2009. 
[36] S. M. Shaahid y M. A. Elhadidy, "Economic analysis of hybrid photovoltaic-diesel-battery power systems for residential loads in hot regions-A step to clean future", Renew. Sustain. Energy Rev., vol. 12, n.o 2, pp. 488-503, 2008.

[37] HOMER, “Homer Energy Software”, 2015. [En línea]. Disponible en: http://www.homerenergy.com/. [Accedido: 01-may-2015].

[38] Steca, "Catálogo de Reguladores e Inversores", 2014, p. 133.

[39] ESMAP, "Technical and economic assessment of offgrid, Mini-grid and grid electrification technologies", en ESMAP technical paper 121/07, 2007, p. 324.
[40] IDEAM, "Atlas de Radiación Solar en Colombia", Cap. 2 y 3. Instituto de Meteorología, Hidrología y Estudios Ambientales- IDEAM, Unidad de Planeación MineroEnergética - UPME, 2005. [En línea]. Disponible en: http://intranet.ideam.gov.co:8080/openbiblio/ Bvirtual/019649/2-RadiacionSolar.pdf. [Accedido: 01-jul-2015]

[41] ISAGEN S.A., S. Botero-Botero, I. Dyner, J. D. Velásquez-Henao, C. J. Franco-Cardona, Y. Benavédes, G. J. Correa-Henao, y J. F. Franco-Barrera, Fuentes no renovables de generación de electricidad. Medellín (Colombia): Universidad Nacional de Colombia, 2005. 